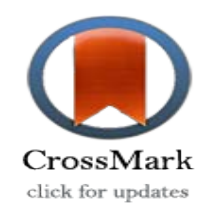

\title{
Prevalence of substance abuse, mental health and frailty among elderly hospitalized in Hazrat-e Rasoul Hospital
}

\section{Ghasem Nasrollahi ${ }^{1}$, Vahid Rashedi ${ }^{2}$, Niloufar Khodabandelou ${ }^{3}$, Mojtaba Habibi ${ }^{4}$, *Behnam Shariati ${ }^{5}$}

1- School of Medicine, Iran University of Medical Sciences, Tehran, Iran.

2- School of Behavioral Sciences and Mental Health (Tehran Institute of Psychiatry), Iran University of Medical Sciences, Tehran, Iran.

3- School of Medicine, Iran University of Medical Sciences, Tehran, Iran.

4- School of Behavioral Sciences and Mental Health (Tehran Institute of Psychiatry), Iran University of Medical Sciences, Tehran, Iran.

5- School of Medicine, Iran University of Medical Sciences, Tehran, Iran (Corresponding Author)

Email: behnamshariatimd@gmail.com

\section{Abstract}

Introduction: People in old age are susceptible to a variety of diseases and disabilities due to decreased physiological capacities. The aim of this study is to investigate the prevalence of substance abuse, mental health, and frailty among the elderly hospitalized in Hazrat-e Rasoul Hospital in 2019.

Methods: This cross-sectional descriptive-analytic study was performed on 300 patients 60 years old and over at Hazrat-e Rasoul Hospital in Tehran. The researcher-made questionnaires for demographics, general health (GHQ), substance use (DAST), and clinical frailty questionnaires (CFS) were used to obtain the data.

Results: The age range of the participants was between 60 and 97 years with a mean of 71 years. $22.7 \%$ equals $68 \%$ of the elderly studied with substance abuse, mean overall health score was 19.78 with a standard deviation of 13.38 and in elderly frailty, good management index with a frequency of 69 persons equals $23 \%$ of Subjects have the highest frequency.

Conclusions: The findings showed that the family, social and economic status of the elderly is influenced by the prevalence of substance abuse and their general health and frailty.

Keywords: Substance abuse, General Health, Frailty, Elderly. 


\title{
بررسى شيوع سوءمصرف مواد، سلامت روان و آسيب يذيرى در سالمندان بسترى در بيمارستان

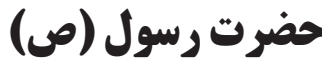

\author{
قاسم نصر اللهى '، وحيد راشدى '، نيلوفر خدابنده لو "، مجتبى حبيبى "، *بهنام شريعتىه
}

1- دانشكده يزشكى، دانشگاه علوم يزشكى ايران، تهران، ايران.

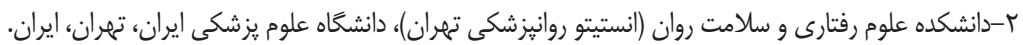

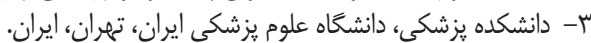

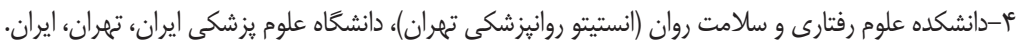

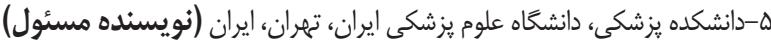
ايميل: behnamshariatimd@gmail.com

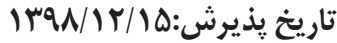

تاريخ دريافت: || || ||

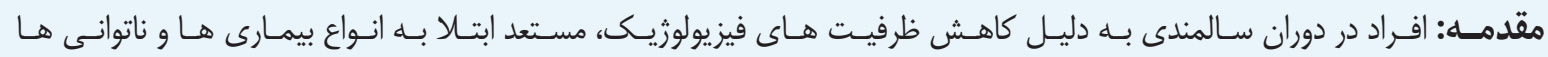

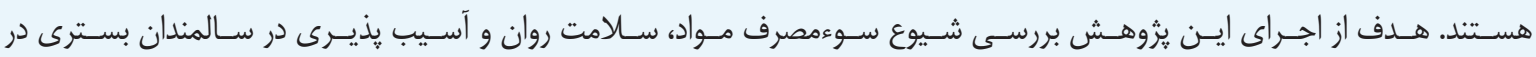
بيمارسـتان حضـرت رسـول (ص) سـال Vو اسـت.

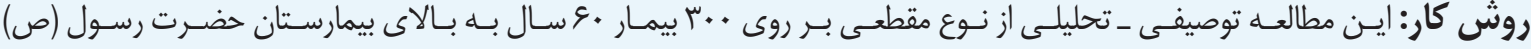

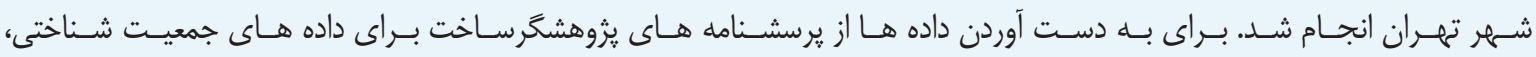

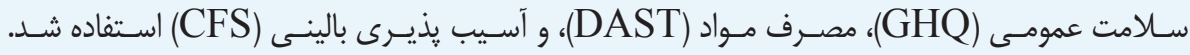

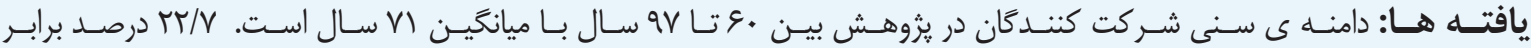

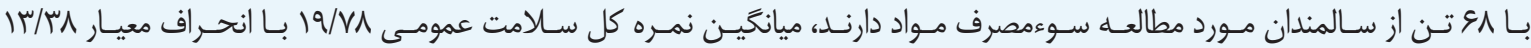

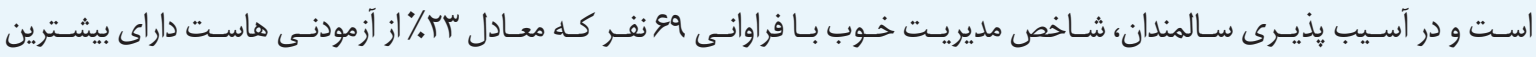
فراوانسى اسـت.

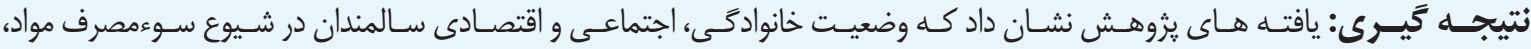

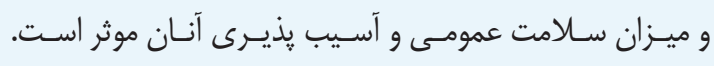
كليد وازه ها: سوءمصرف مواد، سلامت عمومى، آسيب يذيرى، سالمندان.

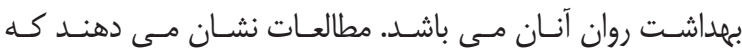
مقلدمه

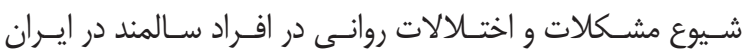

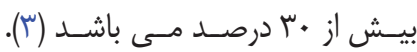

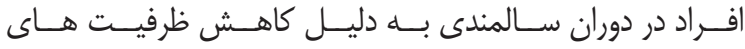

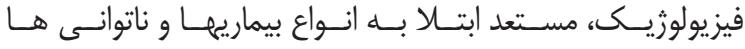

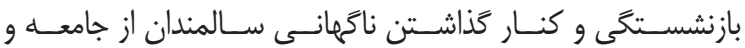

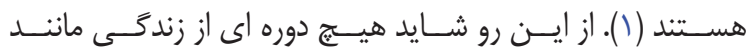
فعاليـت هـاى اجتماعـى در كنـار كاهـش توانايسى عملكـردى آنها،

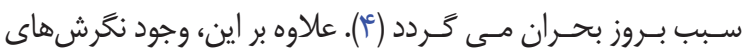
منفى در جامعه و خـانواده نسبت به سالمندان و عدم توجه به تجارب آنـان سـبب تشـديد انزواى اجتماعى و در نهايت كاهش كيفيت

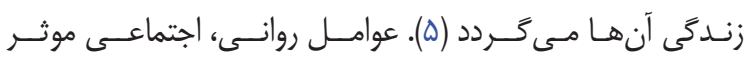

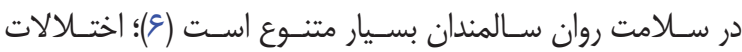

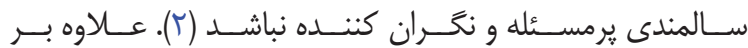
سـامت جســى؛ سـامت روان نيـز شـاخص مهمـى در وضعيـت

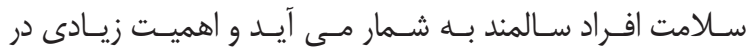

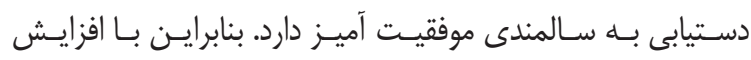

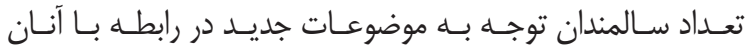
بيشـتر شــه اسـت كــه از جملـهـ ى ايـن موضوعـات، توجـهـ بــهـ 


\section{روش كار}

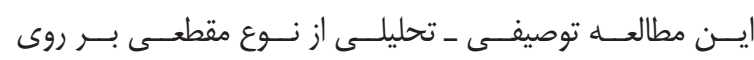

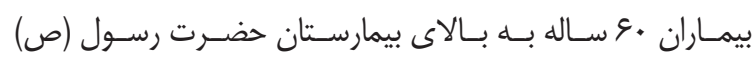

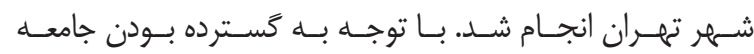

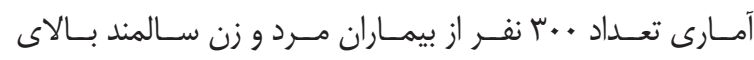

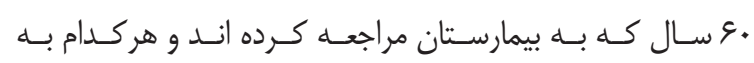

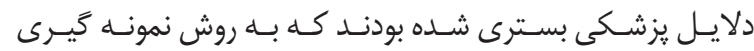
تصادفى سـاده انتخــاب شـــند.

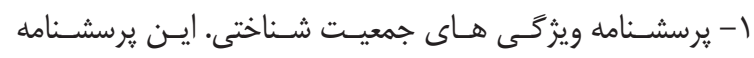

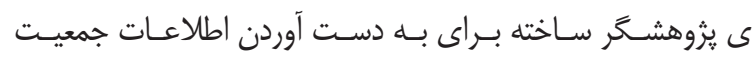

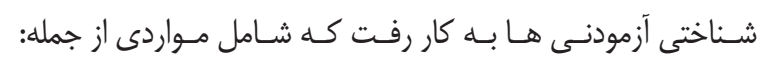

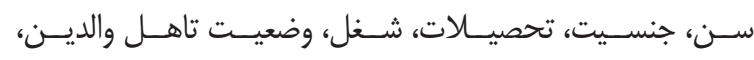
وضعيـت اقتصـادى.

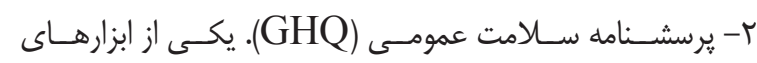
بسـيار معتبــر و ير كاربــرد بــراى ارزيابـى وضعيـت ســلامت در

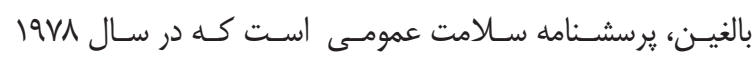

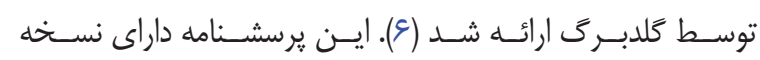

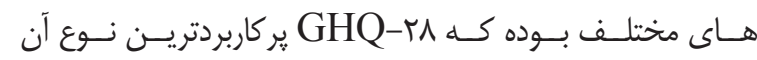

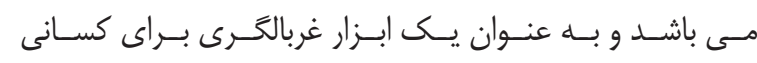

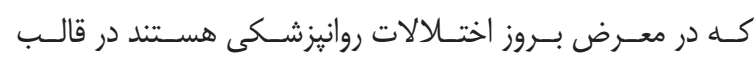

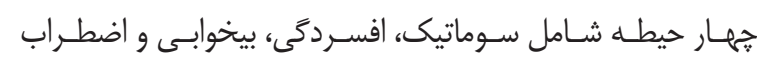

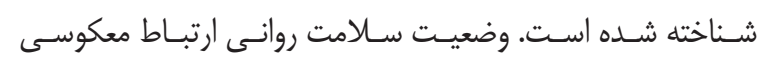

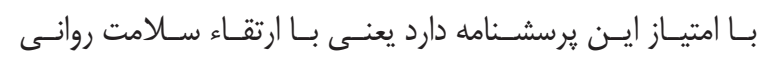

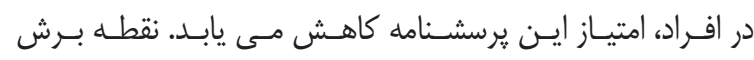

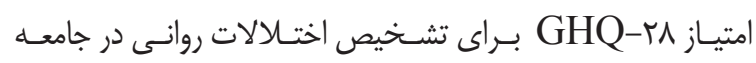

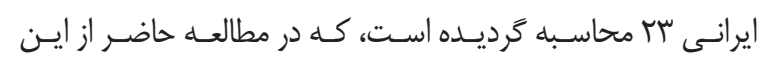
نقطـهـ بــرش اسـتفاده مـى شـود (1). ضريسـب آلفــاى كرونبـاخ

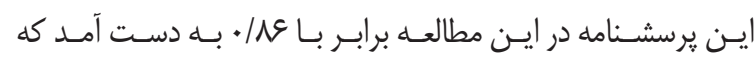

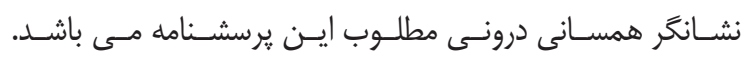

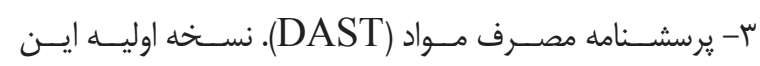

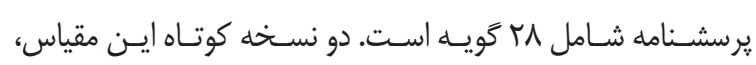

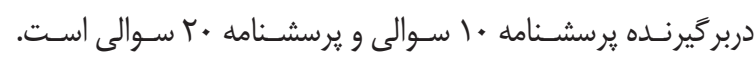

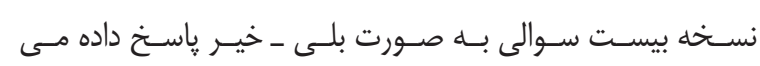

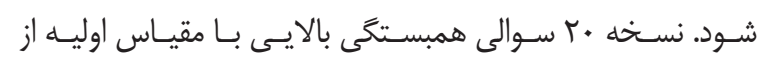

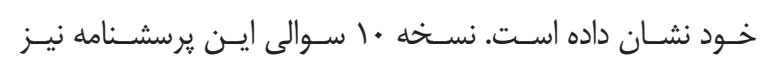

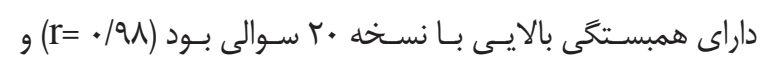

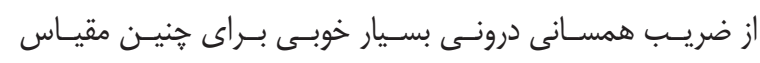

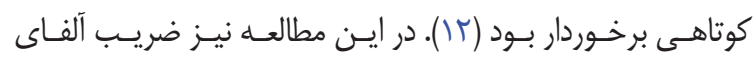

روانشـناختى و ياييـن بــودن سـامت روان در ســالمندان بـهـ دليـل

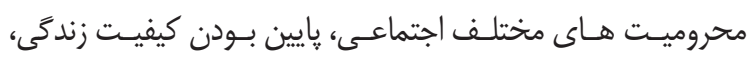

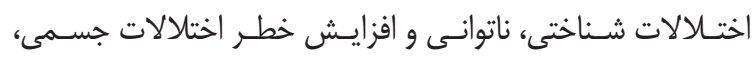

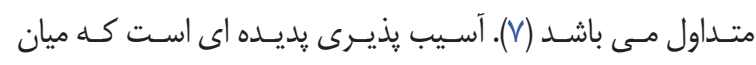

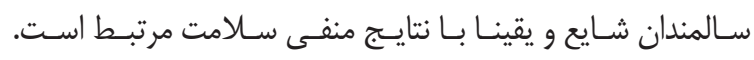

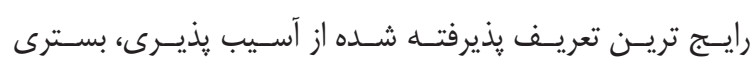

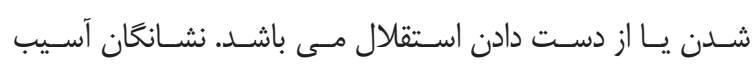

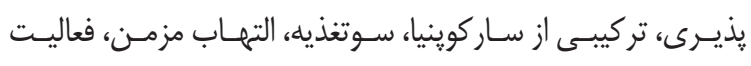

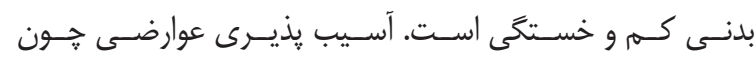

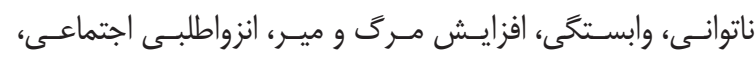

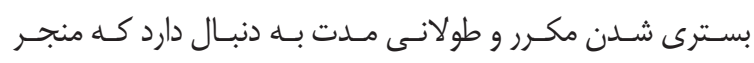

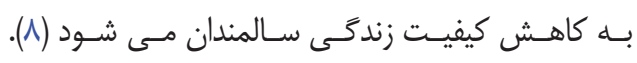

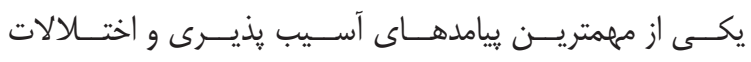

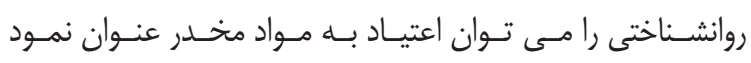

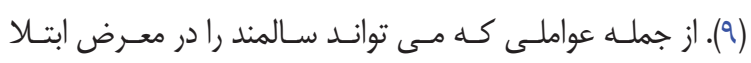

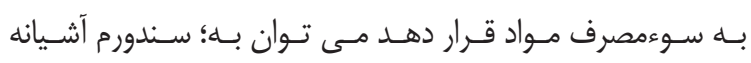

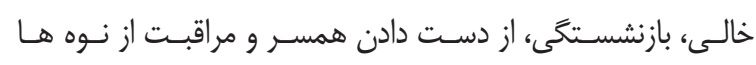

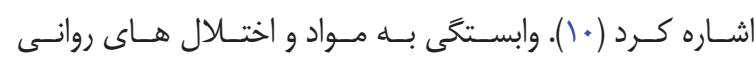

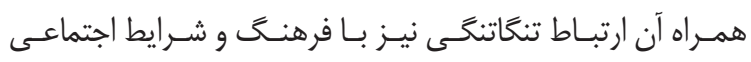

و اقتصـادى هـــر جامعـه دارد (().

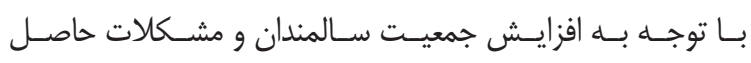

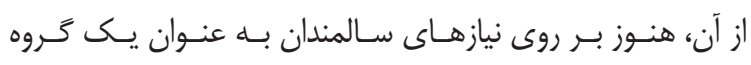

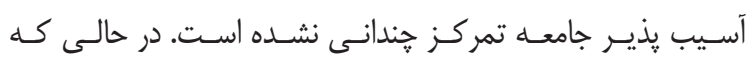

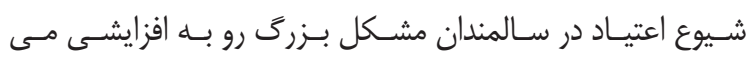

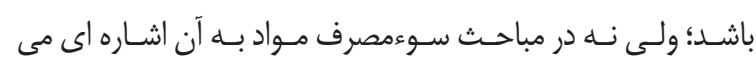

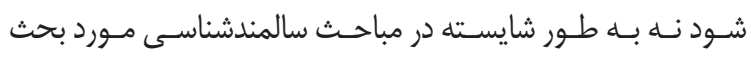

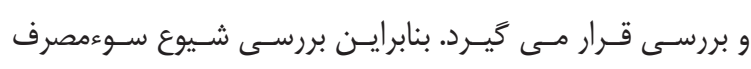

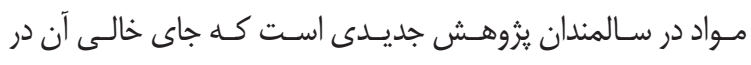

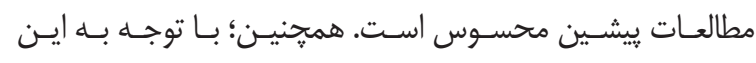
كـهـ عـدم موفقيـت اثربخشـى درمـان هـاى سـنتى در مبتلايـان

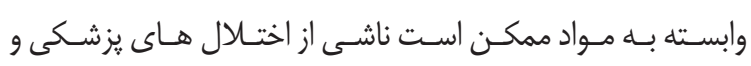

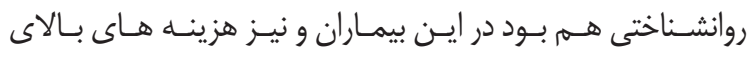

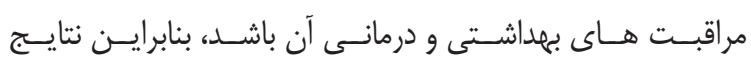

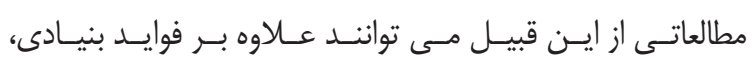

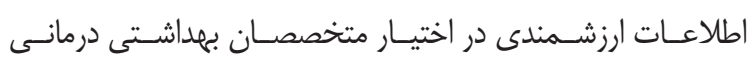

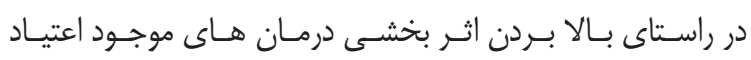
بحَـذارد. 


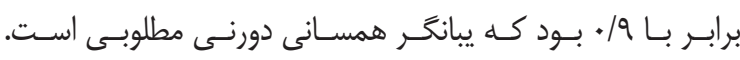

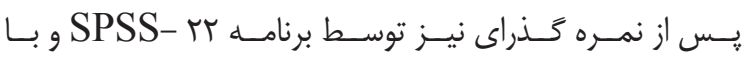

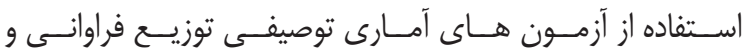

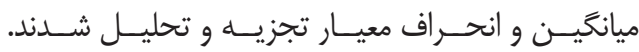

يافته ها

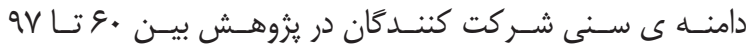

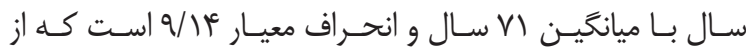

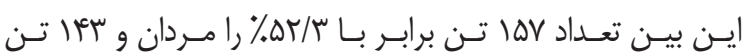

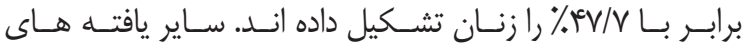

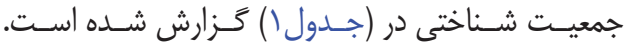

كرونبـاخ يرسشـنامه برابـر بـا هو/ • بــه دسـت آمــد.

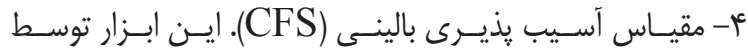

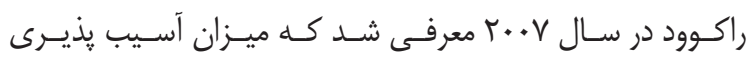

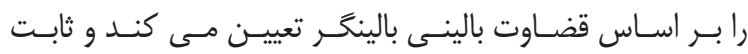

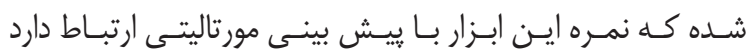

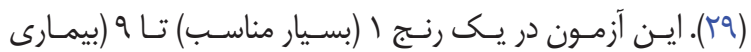

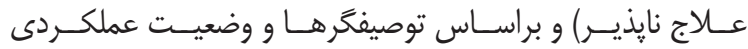

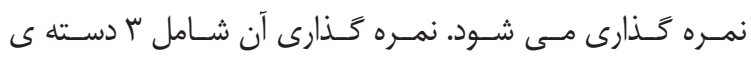

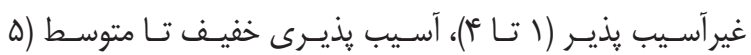

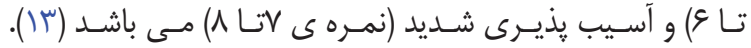

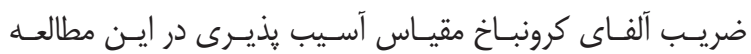

\begin{tabular}{|c|c|c|c|}
\hline \multicolumn{2}{|c|}{$N=\mu .}$. & \multirow{2}{*}{\multicolumn{2}{|c|}{ متغير }} \\
\hline درصد & فراوانى & & \\
\hline GQ/ & 198 & متأهل & \\
\hline$m e / v$ & $1 \cdot f$ & مجرد & ت. تأهل \\
\hline $9 \Delta / \cdot$ & 190 & با همسر & \\
\hline $19 /$. & $\Delta V$ & تنهيا & \\
\hline $\mid q 1$. & is & با فرزند & وضعيت زندكى \\
\hline 1$) / \mathrm{V}$ & TED & مالى & \\
\hline IN/T & $\Delta \Delta$ & مستأجر & وضعيت مسكن \\
\hline س & pq & شاغل & \\
\hline FT/. & 149 & بازنشسته & وصعيت استعال \\
\hline$r \cdot / V$ & TH & خانه دار & \\
\hline
\end{tabular}

جدول rا: توزيع فراوانى وضعيت سوءمصرف مواد در سالمندان

\begin{tabular}{|c|c|c|c|c|c|c|c|}
\hline \multicolumn{6}{|c|}{$\mathrm{N}=\mu .}$. & \multirow{2}{*}{ فراوانى } & \multirow{2}{*}{ متغير } \\
\hline مجموع & شديد & قابل توجه & متوسط & קֶايين & عدم مصرف & & \\
\hline r.. & r & • & + & 99 & זחז & تعداد & \\
\hline $1 \cdots$ & $\cdot / V$ & • & • & $T r /$. & $V V / \mu$ & درصد & وصعيت سوء \\
\hline
\end{tabular}

تعـداد يـــ مــورد سـوءمصرف هروئيسن و باقـى افـراد اعتيـاد بــه

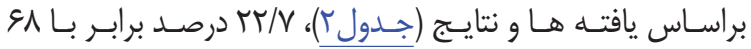
تريـاك بــه شـكل خوراكـى و استنشـاقى دارنـد. تـن از سـالمندان مـورد مطالعـه سـوءمصرف مـواد دارند كـهـ از اين

جدول س: توزيع شاخص هاى مركزى و ير اكندگى سلامت عمومى در سالمندان

\begin{tabular}{|c|c|c|c|c|}
\hline \multicolumn{4}{|c|}{$N=\mu \ldots$} & \multirow{2}{*}{ متغير } \\
\hline انحر اف معيار & حداكثر & حداقل & ميانگين & \\
\hline سז/ץ & 15 & . & $r / v$. & نشانه هاى جسماني \\
\hline$\Delta / \cdot r^{c}$ & 10 & • & $f / \kappa$. & اضطراب و بى خوابى \\
\hline t/AF & 10 & $\Delta$ & N/DH & اختلال در كاركرد اجتماعى \\
\hline عس/س & 15 & - & $r / T f$ & افسردگى \\
\hline 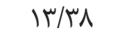 & $\Delta 1$ & $\varepsilon$ & $19 / 11$ & نمره كل سلامت عمومى \\
\hline
\end{tabular}

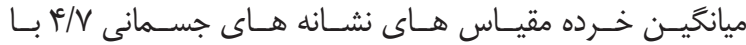

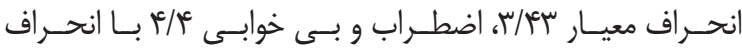

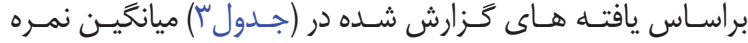
كل سـامت عمومـى 19/V1 بــا انحــراف معيـار رس/سا اسـت. 
جدول f: توزيع فراوانى شاخص آسيب يذيرى در سالمندان

\begin{tabular}{|c|c|c|c|c|c|c|c|c|c|c|c|}
\hline \multicolumn{10}{|c|}{$\mathrm{N}=\mu .}$. & \multirow[b]{2}{*}{ فراوانى } & \multirow[b]{2}{*}{ متغير } \\
\hline مجموع & انتهايى & شيديرى & شذيرى & يذّيرى & خذيفي & لِّير & خديريت & مناسب & مناسبار & & \\
\hline $\begin{array}{l}r . . \\
1 . .\end{array}$ & . & . & $\begin{array}{l}F \% \\
H \%\end{array}$ & $\begin{array}{l}r v \\
T / r / r\end{array}$ & $\begin{array}{l}\Delta \Delta \\
N / r\end{array}$ & $\begin{array}{l}G r \\
r \cdot / V\end{array}$ & $\begin{array}{l}99 \\
r \% / .\end{array}$ & $\begin{array}{l}\mu c \\
11 / \mu\end{array}$ & $\begin{array}{c}1 \\
. / \mu\end{array}$ & تعرداد & آسيب باخيرى \\
\hline
\end{tabular}

زن، انـزواى اجتماعىى، سـابقه ى مصــرف مــواد يـا اختـلال در

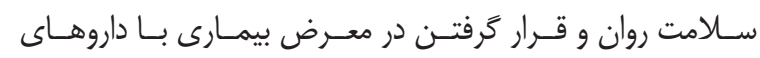

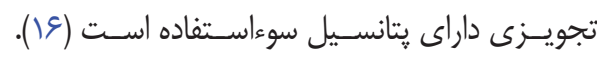

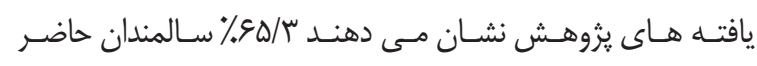

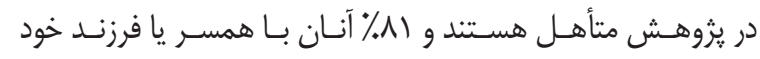
زندكَى مسى كنتـد. داشـتن همســـ در سـنين سـالمندى مـى تواند

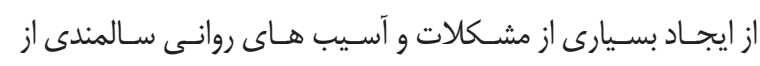

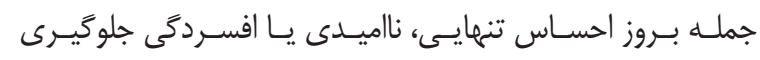

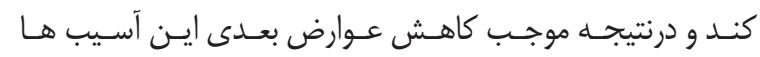

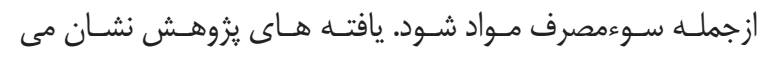

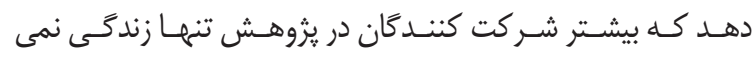

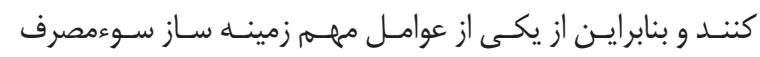

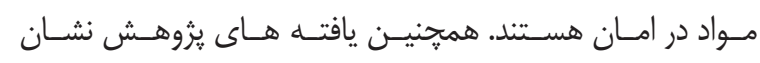

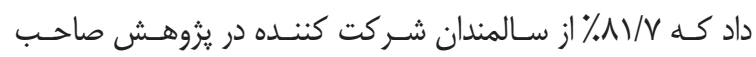

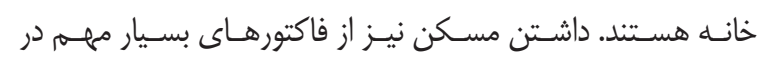

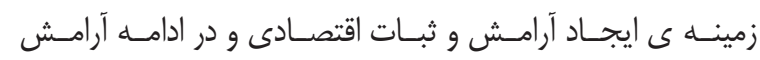

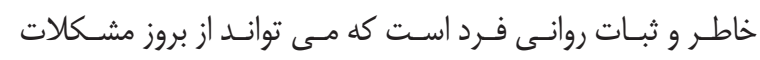

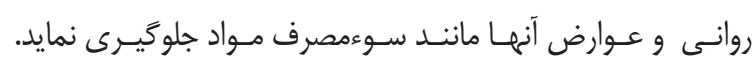

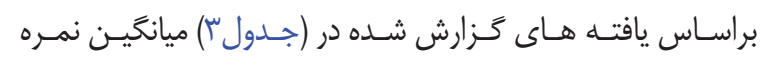

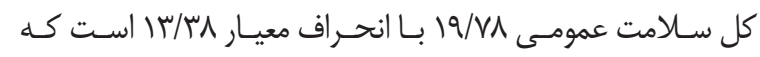

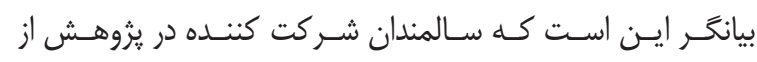

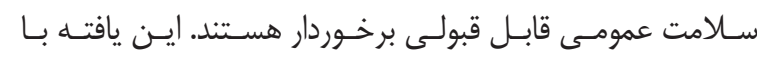

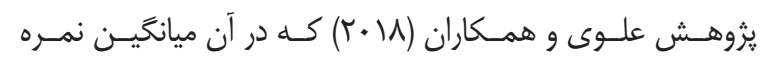

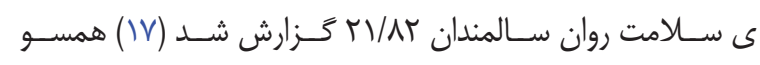

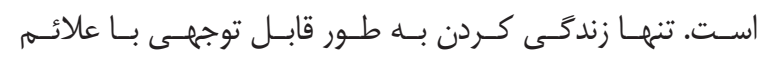

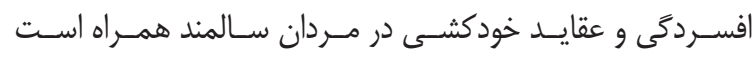

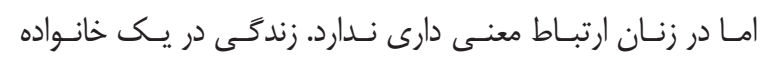

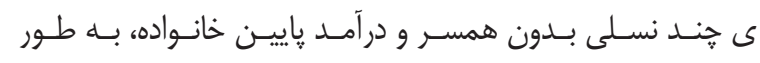

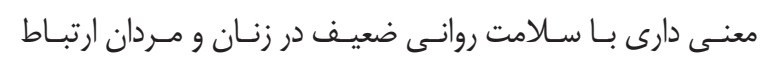

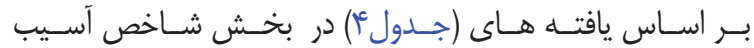

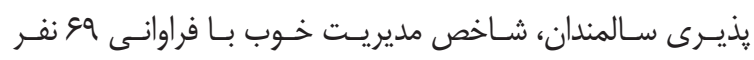

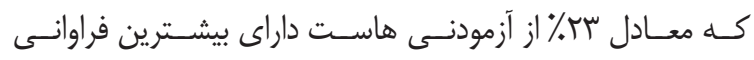

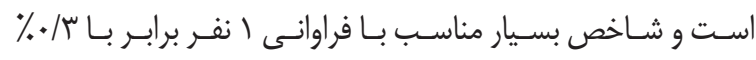

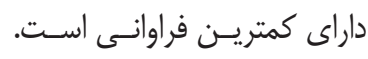

\section{بحث و نتيجه كيرى}

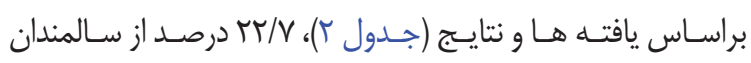

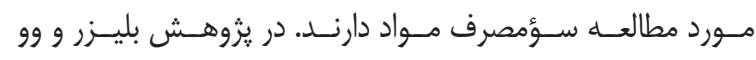

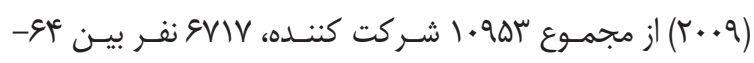

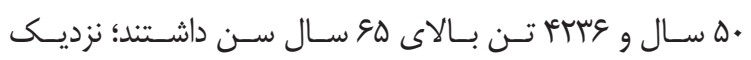

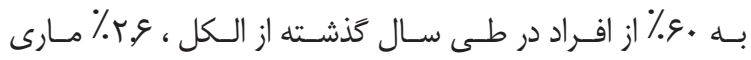

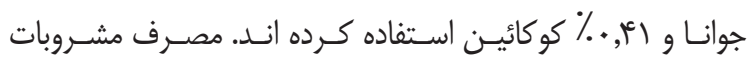

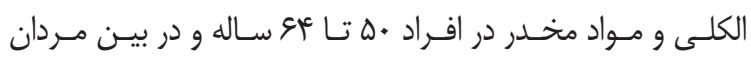

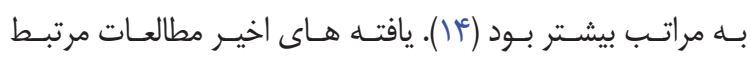

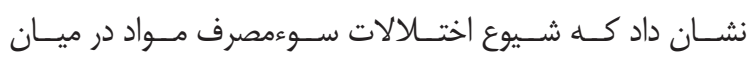

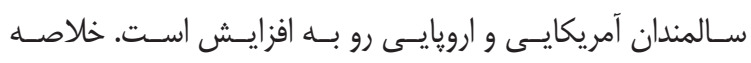

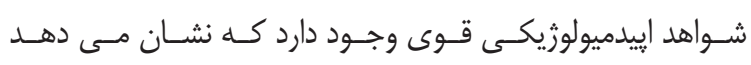

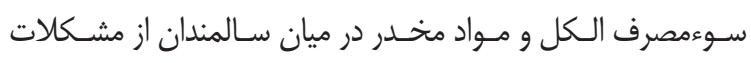

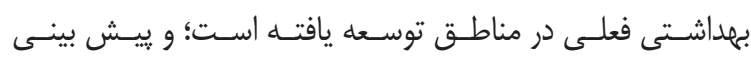

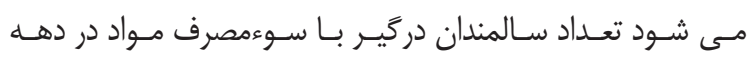

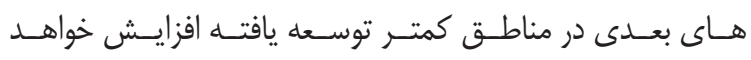

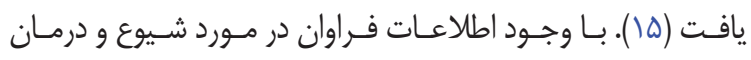

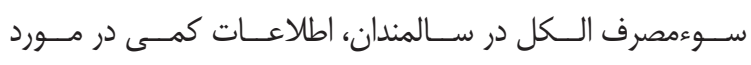

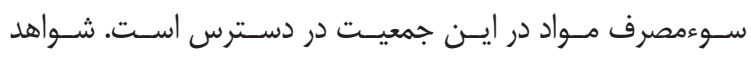

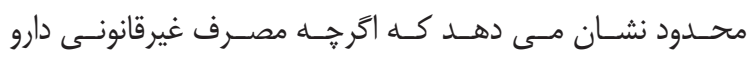

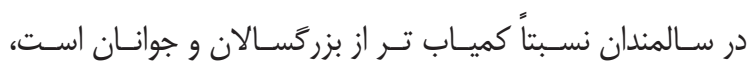

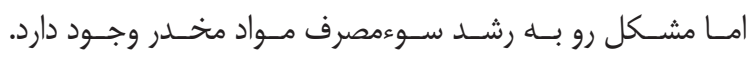

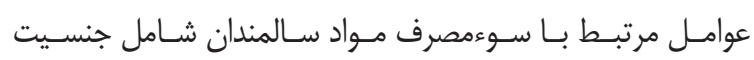


كيرشناسـى در كشـورهاى گَوناتـون نيز آسـيب يذيـرى در جمعيت

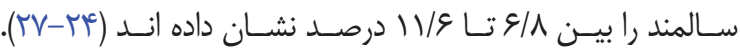

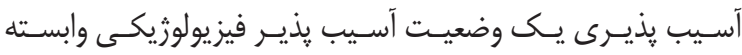

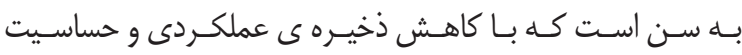

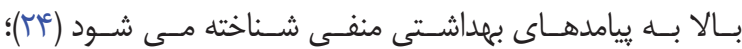

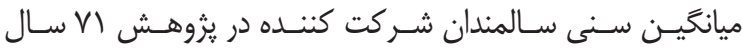

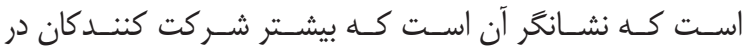

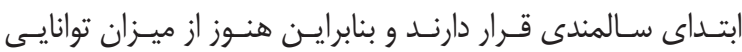

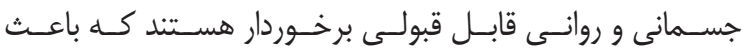

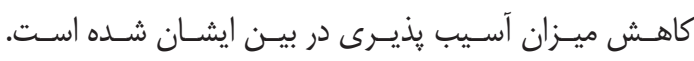

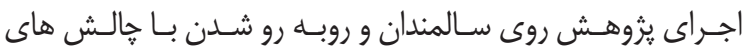

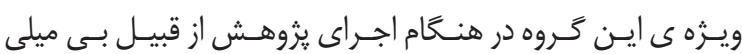

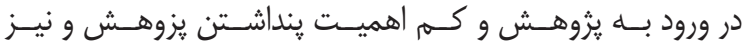

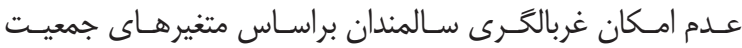

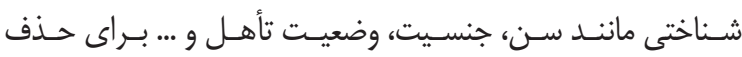

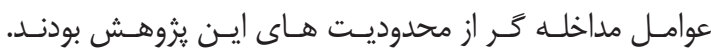

\section{تشكر و قدردانى}

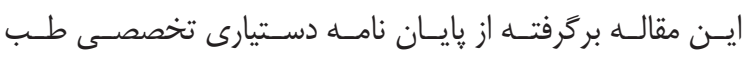

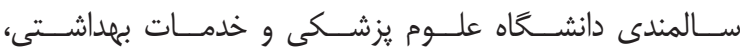

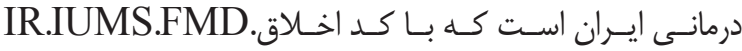

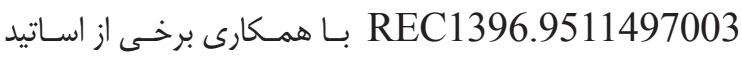

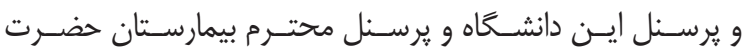

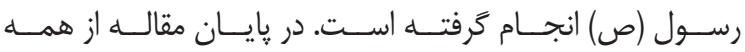

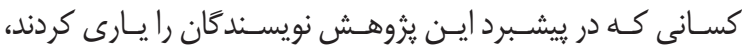

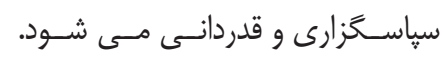

\section{Refrences}

1. Kheirkhah F, Hosseini SR, Fallah R, Bijani A. Prevalence of cognitive disorders in elderly people of Amirkola (2011-2012). Iranian Journal of Psychiatry and Clinical Psychology. 2014; 19 (4): 247-54.

2. Sajadi H, Mohaqeqi Kamal H, Vameghi M, Forozan AS, Rafei H, Nosratabadi M. Systematic review of prevalence and risk factors associated with depression and its treatment in Iranian elderly. Iranian Journal of Ageing. 2013; 7 (4):715.

3. Salar A, Boryri T, Khojasteh F, Salar E, Jafari H, Karimi M. Evaluating the physical, psychological and social problems and their

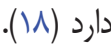

اختـلالات اضطرابــ شـايع تريـن اختـلالات روانشـناختى دوران

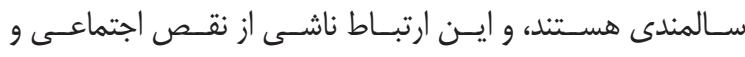

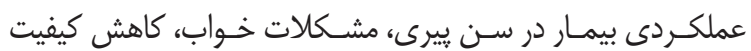
زندگى مـى باشـــ (19). افزايـش روابـط اجتماعى و تقويـت تعامل موجـب كاهش احسـاس تنهايسى و اضطــاب جدايى در سـالمندان

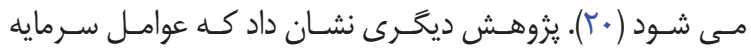

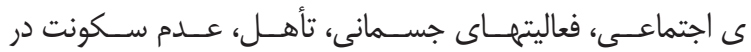

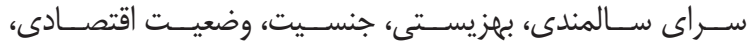
تحصيـلات و شـيوه هـــاى مختلــف درمانسى بــر سـلامت روان

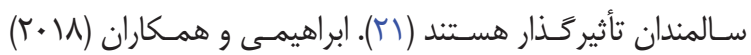

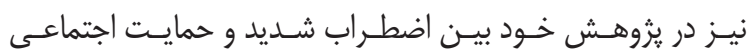

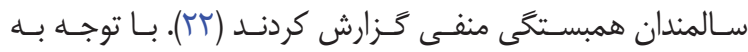

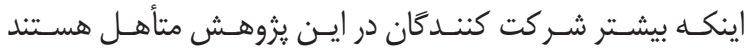

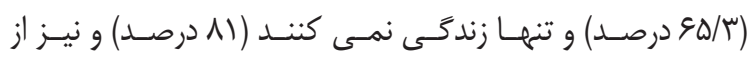

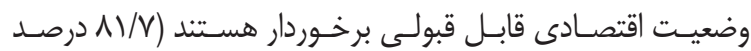
صاحسب خانـه هسـتند)، مسى تـوان تصـور كـرد كـه عوامـل زمينه

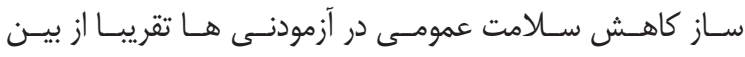

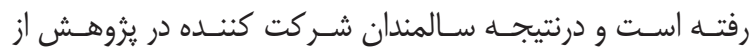
سـامت عمومسى نسـبتا خوبـى برخـودار هســتند.

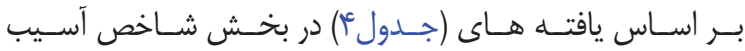

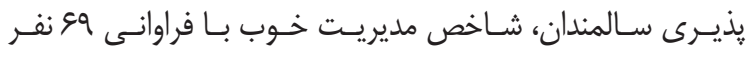

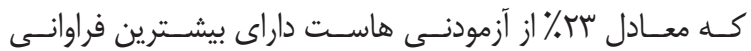

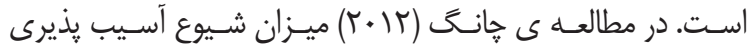

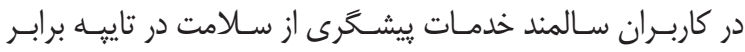

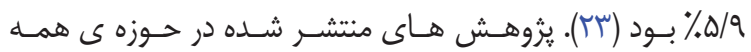

relation to demographic factors among the elderly in Zahedan city during 2010-2012. KAUMS Journal (FEYZ). 2013; 17 (3): 305-11.

4. Kiefer RA. An integrative review of the concept of well-being. Holistic Nursing Practice. 2008; 22 (5): 244-52.

5. Larkin M. Robert Butler: Championing a healthy view of ageing. The Lancet. 2001; 357(9249):48.

6. Roudsari MS, Nedjat S, Foroughan M, Shahboulaghi FM, Rashedi V, Haghi M, Chehrehnegar N, Mansouri T. Protective psychosocial factors of geriatric depression in community-dwelling older adults: A review article. Iranian Journal of Psychiatry and Behavioral Sciences. 2018; 12 (2):e10652. 
7. Negahban Z, Arab M, Tajvar M, Rahimi Fa, Rashidyan A. To Investigate The Association Between Social Capital And Mental Health In The Ageing Population Of Tehran. 2015.

8. Dent E, Visvanathan R, Piantadosi C, Chapman I. Use of the Mini Nutritional Assessment to detect frailty in hospitalised older people. The journal of nutrition, health \& aging. 2012;16 (9): 764-7.

9. Petrakis IL, Gonzalez G, Rosenheck R, Krystal JH. Comorbidity of Alcholism and Psychiatric Disorders: An Overview. Alcohol Research. 2002; 26 (2):81.

10. Nodehi Moghadam A, Ehsani Fard F. Prevalence of Physical Impairment Among the Elderlly Persons of the Tehran City in 1385. Iranian Journal of Ageing. 2007;1(2):125-31.

11. Najafi B, Arzaghi M, Fakhrzadeh H, Sharifi F, Shoaei S, Alizadeh M, et al. Mental health status and related factors in aged population: Urban health equity assessment and response tool (Urban-HEART) study in Tehran. Iranian Journal of Diabetes and Metabolism. 2013;13(1):62-73.

12. Alitabar SHS, Falahatpisheh M, Asgarabad MH, Arvin M, Sarvestani A. Psychometric properties of the problems assessment for substance using psychiatric patients. Community Health (Salāmat-i ijtimāī). 2016; 3 (1):11-20.

13. Juma S, Taabazuing M-M, Montero-Odasso M. Clinical frailty scale in an acute medicine unit: a simple tool that predicts length of stay. Canadian Geriatrics Journal. 2016;19 (2): 34.

14. Blazer DG, Wu L-T. The epidemiology of substance use and disorders among middle aged and elderly community adults: national survey on drug use and health. The American Journal of Geriatric Psychiatry. 2009; 17 (3):237-45.

15. Wang Y-P, Andrade LH. Epidemiology of alcohol and drug use in the elderly. Current opinion in psychiatry. 2013; 26 (4):343-8.

16. Simoni-Wastila L, Yang H-WK. Drug abuse and addiction in elderly. Drug Abuse and Addiction in Medical Illness: Springer; 2012. p. 455-65.

17. Alavi M, Jorjoran Shushtari Z, Noroozi M, Mohammadi Shahboulaghi F. Mental health and related factors in old population in Tehran 2014-2015. Journal of Mazandaran University of Medical Sciences. 2018; 27 (158): 112-22.

18. Jeon G-S, Jang S-N, Rhee S-J, Kawachi I, Cho S-I. Gender differences in correlates of mental health among elderly Koreans. The Journals of Gerontology Series B: Psychological Sciences and Social Sciences. 2007;62 (5): S323-S9.

19. Ruppert PD, Attix DK, Pachana N, Laidlaw K. Evaluation and Treatment of Geriatric Neurocognitive Disorders. The Oxford Handbook of Clinical Geropsychology: OUP Oxford; 2014. p. 285.

20. Khademi MJ, Rashedi V, Sajadi S, Gheshlaghi SM. Anxiety and loneliness in the Iranian older adults. International Journal of Psychology and Behavioral Sciences. 2015; 5 (2):49-52.

21. Arabzadeh M. Meta-analysis of Effective Factors in Mental health of older people. 2016.

22. Ebrahimi B, Hosseini M, Rashedi V. The Relationship between Social Support and Death Anxiety among the Elderly. Elderly Health Journal. 2018; 4 (2): 37-42.

23. Chang Y-W, Chen W-L, Lin F-G, Fang W-H, Yen M-Y, Hsieh C-C, et al. Frailty and its impact on health-related quality of life: a crosssectional study on elder community-dwelling preventive health service users. PloS one. 2012;7 (5):e38079.

24. Fried LP, Tangen CM, Walston J, Newman AB, Hirsch C, Gottdiener J, et al. Frailty in older adults: evidence for a phenotype. The Journals of Gerontology Series A: Biological Sciences and Medical Sciences. 2001; 56 (3): M146-M57.

25. Bandeen-Roche K, Xue Q-L, Ferrucci L, Walston J, Guralnik JM, Chaves P, et al. Phenotype of frailty: characterization in the women's health and aging studies. The Journals of Gerontology Series A: Biological Sciences and Medical Sciences. 2006; 61(3): 262-6.

26. Frisoli Jr A, Chaves PH, Ingham SJM, Fried LP. Severe osteopenia and osteoporosis, sarcopenia, and frailty status in community-dwelling older women: results from the Women's Health and Aging Study (WHAS) II. Bone. 2011;48 (4): 952-7.

27. Lin C-C, Li C-I, Chang C-K, Liu C-S, Lin C-H, Meng N-H, et al. Reduced health-related quality of life in elders with frailty: a cross-sectional study of community-dwelling elders in Taiwan. PloS one. 2011;6 (7):e21841. 